\title{
A CONTRARIO DETECTION OF GOOD CONTINUATION OF POINTS
}

\author{
José Lezama ${ }^{\star \dagger}$ Rafael Grompone von Gioi ${ }^{\star} \quad$ Gregory Randall $^{\dagger} \quad$ Jean-Michel Morel ${ }^{\star}$ \\ * CMLA, ENS Cachan, France \\ †Universidad de la República, Uruguay
}

\begin{abstract}
We will consider the problem of detecting configurations of points regularly spaced and lying on a smooth curve. This corresponds to the notion of good continuation introduced in the Gestalt theory. We present a robust algorithm for clustering points along such curves, whilst at the same time discarding noisy samples. Based on the a contrario methodology, the detector builds upon a simple, symmetric primitive for a triplet of points, and finds statistically meaningful chains of such triplets. An efficient implementation is proposed using the Floyd-Warshall algorithm. Experiments on synthetic and real data show that the method is able to identify the perceptually relevant configuration of points in good continuation.
\end{abstract}

Index Terms - good continuation detection, points, curves, Gestalt, a contrario

\section{INTRODUCTION}

The Gestalt school of psychology [1, 2, 3, 4] proposed in the 1920 s a theory of perception based on a list of geometric grouping laws that govern the process of visual perception. The theory implies that the sensory data is hierarchically grouped into a global interpretation of the scene. One of these laws, the so-called good continuation principle, can be stated as "All else being equal, elements that can be seen as smooth continuations of each other tend to be grouped together" [5]. In the example in Fig. 1 this law would result in a three part organization: a line, an arc of circle, and a zigzag, all formed by dots. Unfortunately, these laws were enunciated qualitatively; the theory gives no precise prediction of how the perceptual organization would change if the dots of the figure are gradually displaced.

D. Lowe was among the first to state the importance of incorporating the Gestalt principles of colinearity, cocurvilinearity and simplicity for perceptual grouping in the computational domain [6]. Some formalizations of the good continuation principle determine a "saliency map" of an image of dot patterns or edge elements [7, 8]. [8] introduces the tensor voting strategy, a successful approach that was continued in various work [9, 10]. A statistical analysis of curves of points under random noise was carried out in $[11,12]$. A pyramidal model for good continuation was proposed in [13],

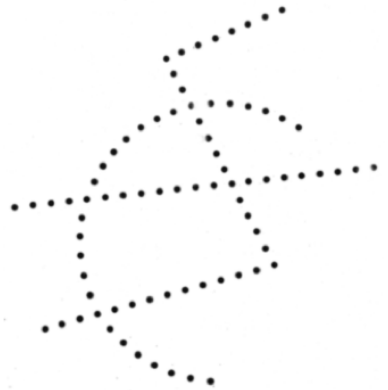

Fig. 1. Good Continuation law: human perception tends to group elements on a smooth, continuous order. Image extracted from [2].

were a psychophysical study was conducted and the effect of dot density, length, and curvatures was analyzed.

This work proposes an unsupervised method for detecting groups of points in good continuation. The a contrario framework [14] is used to provide automatic detection thresholds, compatible with perception, and to handle noise points. The main idea is the non-accidentalness principle, which states that only configurations unlikely to appear just by chance are relevant. A previous work [15] proposed an a contrario method for the related but different problem of detecting good continuations of image edges.

Given a set of points in a domain, we will consider each possible chain of points as a candidate for good continuation. We will call triplets the sets of three consecutive points in the chain. In a perfect good continuation chain, each triplet would have the third point as the symmetric of the first w.r.t. the second. The precision of a triplet will be measured by the distance from the observed third point, to its ideal position, see Fig. 2 (left). The whole chain will be characterized by the number of triplets and its worst precision. A chain is said to be in good continuation when one would rarely observe such precision if the points were randomly distributed (i.e., organized "just by chance"). Testing every possible chain of points would result in a combinatorial explosion; instead, the candidates are selected using a heuristic approximation.

This paper is organized as follows: Section 2 presents the a contrario model of good continuation chains and Sect. 3 describes an efficient implementation. The algorithm is then 


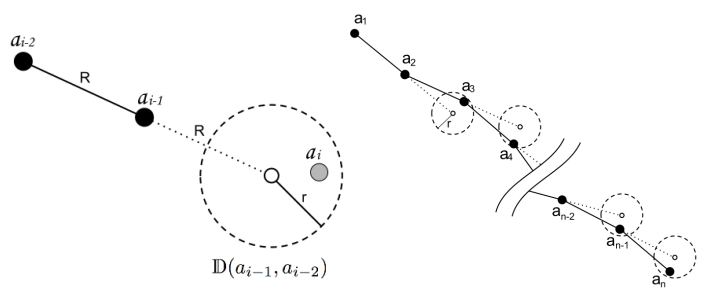

Fig. 2. Definition of the good continuation event. Left: we shall say that a triplet of points $a_{i-2}, a_{i-1}, a_{i}$ is in good continuation when $a_{i}$ lies within the disc $\mathrm{D}_{r}\left(a_{i-1}, a_{i-2}\right)$, centered in the symmetric of $a_{i-2}$ with respect to $a_{i-1}$ and with radius $r$. Right: we shall say that a chain of $n$ points is in good continuation if for each $i \geq 3, a_{i} \in \mathrm{D}_{r}\left(a_{i-1}, a_{i-2}\right)$.

evaluated in Sect. 4 on synthetic and real data. Finally, Sect. 5 concludes the paper.

\section{MATHEMATICAL MODEL}

Let us consider a configuration of three points $a_{i-2}, a_{i-1}$ and $a_{i}$, in the $[0,1] \times[0,1]$ square such as the one shown in the left part of Fig. 2. Let us call $\mathrm{D}_{r}\left(a_{i-1}, a_{i-2}\right)$ the disc centered in the symmetric point of $a_{i-2}$ with respect to $a_{i-1}$ and with radius $r$. We shall call a triplet of points in good continuation (up to a precision $r$ ), a triplet where the third point $a_{i}$ lies in the disc $\mathrm{D}_{r}\left(a_{i-1}, a_{i-2}\right)$. We will consider a chain $\mathcal{C}$ of $n$ points $a_{1}, a_{2}, \ldots, a_{n}$, to be in good continuation (up to a precision $r$ ) if for each $i \geq 3, a_{i} \in \mathrm{D}_{r}\left(a_{i-1}, a_{i-2}\right)$ as shown in the right part of Fig. 2.

We will now use the non-accidentalness principle as explained by D. Lowe: "we need to determine the probability that each relation in the image could have arisen by accident, $P(a)$. Naturally, the smaller that this value is, the more likely the relation is to have a causal interpretation" [6, p. 39]. Our random or a contrario model $H_{0}$, used to evaluate accidentalness, is a set of $N$ independent points, drawn from a uniform distribution on the domain. Under this hypothesis, let us consider the probability $\mathrm{P}(\mathcal{C})$ of having a chain $\mathcal{C}$ of $n$ points in the $[0,1] \times[0,1]$ square in a good continuation configuration. We can write this probability as:

$$
\begin{gathered}
\mathrm{P}(\mathcal{C})=\mathrm{P}\left(a_{3} \in \mathrm{D}_{r}\left(a_{2}, a_{1}\right), a_{4} \in \mathrm{D}_{r}\left(a_{3}, a_{2}\right),\right. \\
\left.\ldots, a_{n} \in \mathrm{D}_{r}\left(a_{n-1}, a_{n-2}\right)\right)= \\
=\int_{a_{1}} \int_{a_{2}} \cdots \int_{a_{n}} 1_{a_{3} \in \mathrm{D}_{r}\left(a_{2}, a_{1}\right)} 1_{a_{4} \in \mathrm{D}_{r}\left(a_{3}, a_{2}\right)} \\
\ldots 1_{a_{n} \in \mathrm{D}_{r}\left(a_{n-1}, a_{n-2}\right)} d a_{1} d a_{2} \ldots d a_{n} .
\end{gathered}
$$

By the definition of $\mathrm{D}_{r}$, we know that, in each integration step,

$$
\int_{a_{i}} 1_{a_{i} \in \mathrm{D}_{r}\left(a_{i-1}, a_{i-2}\right)} d a_{i} \leq \pi r^{2},
$$

where the less or equal sign is to account for the fact that portions of the circles could fall outside the $[0,1] \times[0,1]$ domain.
Finally,

$$
\mathrm{P}(\mathcal{C}) \leq\left(\pi r^{2}\right)^{n-2}
$$

The fundamental quantity in the a contrario methodology is the Number of False Alarms or NFA of an event, defined as the number of tests, times the probability of the event. The NFA for a chain of points in good continuation is computed as:

$$
\operatorname{NFA}(\mathcal{C})=N_{\text {tests }} \cdot \mathrm{P}(\mathcal{C})
$$

The number of tests $N_{\text {tests }}$ is the number of possible chains of points in an image with $N$ points. The number of possible groups of $n$ points among $N$, without repeating a point is $\frac{N !}{(N-n) !}$. Also, if we consider every possible length $n$ from 1 to $N$, the number of possible configurations is approximately $N \times \frac{N !}{(N-n) !}$. Finally, the NFA of the event "having $n$ points in good continuation configuration up to a precision given by the radius $r$ " is then:

$$
\mathrm{NFA}=N \times \frac{N !}{(N-n) !} \cdot\left(\pi r^{2}\right)^{n-2} .
$$

Given an observed set of $N$ points and a candidate chain of $n$ points, we will consider the latter event as a $\varepsilon$-meaningful good continuation when the corresponding NFA value is lower than $\varepsilon$. It can be shown [14] that the expected number of events with NFA $<\varepsilon$ is bounded by $\varepsilon$ in the a contrario model $H_{0}$. This justifies the definition and name of the NFA, as it controls the average number of accidental (thus false) detections. Following [14], we will usually set $\varepsilon=1$.

Note that there is a maximum radius $r$ that can lead to $\varepsilon$-meaningful detections; in the limit case, all the $N$ points participate in a chain with $\mathrm{NFA}=\varepsilon$. In practice, this upper bound on $r$ will allow the algorithm to reduce its computations by discarding all triplets that would never be part of a valid chain. Substituting $n=N$ in eq. 8 we get

$$
r_{\max }(\varepsilon)=\sqrt{\frac{1}{\pi}\left(\frac{\varepsilon}{N \times N !}\right)^{\frac{1}{N-2}}} .
$$

\section{ALGORITHM DESCRIPTION}

This section describes an efficient implementation of the method presented in the previous section. Algorithm 1 presents a heuristic based on the Floyd-Warshall algorithm to compute $\varepsilon$-meaningful good continuation chains. Algorithm 2 describes a redundancy reduction step.

For the sake of limiting the complexity, the algorithm will consider only triplets formed by points and their $K$ nearest neighbors ${ }^{1}$, and whose precision is lower than the maximum obtained with Equation (9). This is the softest bound for a triplet, since for shorter chains, a higher precision is required. Lines 1 to 11 of Algorithm 1 build the list $\mathcal{T}$ of triplets to

\footnotetext{
${ }^{1}$ The results shown in this article use $K=4$.
} 


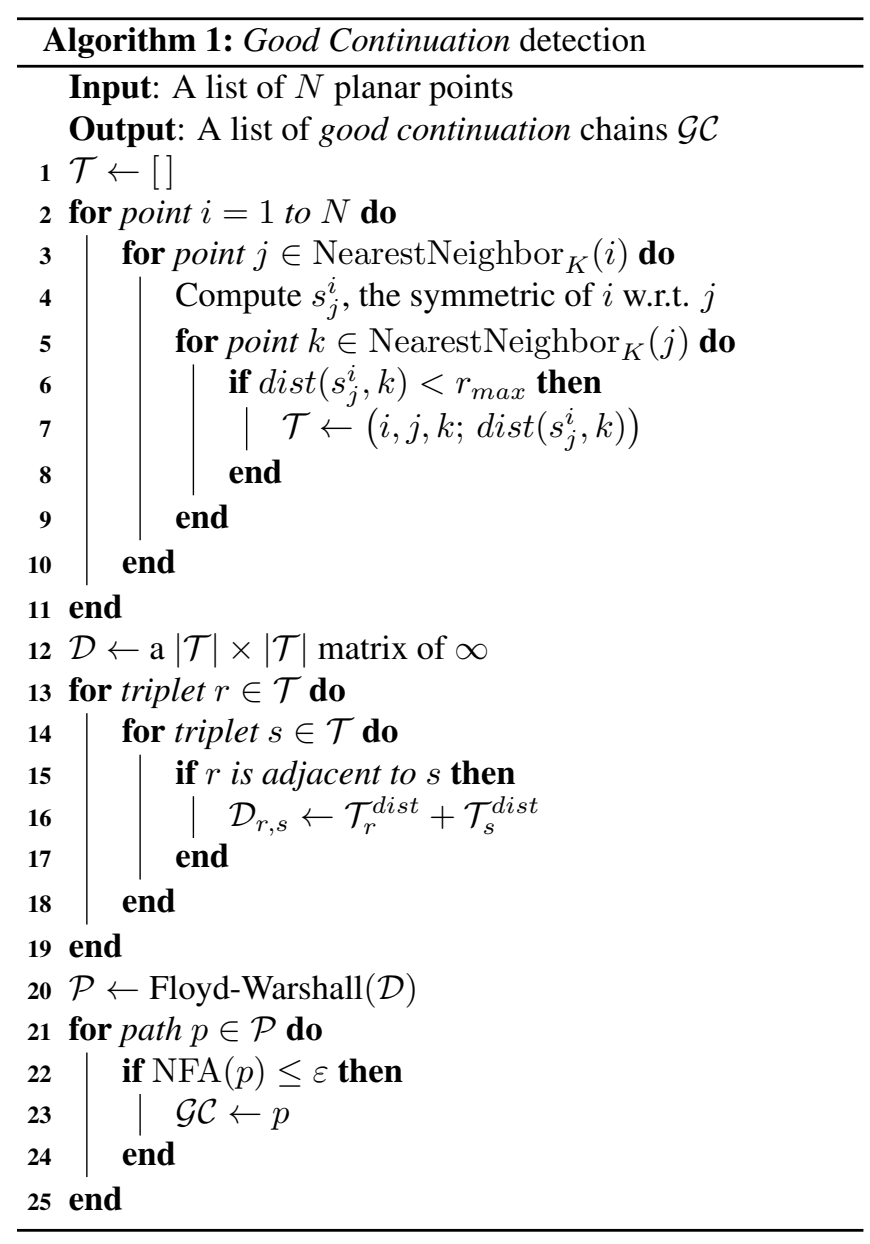

be considered. Note that each triplet is stored with the radius $\operatorname{dist}\left(s_{j}^{i}, k\right)$ that measures its precision.

A pair of triplets will be called adjacent when they share two points in such a way that they can form a chain of four points. (Triplets that share two points but form a "Y" shape are not adjacent.) We define a graph where triplets are the vertices and adjacent triplets share an edge with value $r_{s}+r_{r}$. Lines 12 to 19 compute the distance matrix $\mathcal{D}$ that defines this graph.

Once the adjacencies are determined, our method uses the Floyd-Warshall algorithm to compute the path with shortest distances between every two vertices. This will provide the best chain going from every pair of triplets, in the sense of the smallest sum $\sum_{i} r_{i}$ along the path. This does not necessarily correspond to the smallest NFA value; thus, it may not provide the best solution, but in general it would get a satisfactory one. In this sense, this is a heuristic to obtain candidate chains.

The Floyd-Warshall algorithm provides the whole solution in $O\left(|V|^{3}\right)$, where $|V|$ is the number of vertices in the graph, i.e., the number of triplets. The result is a non-linear algorithm, but fast enough to be used in practice. Finally, all the candidate chains provided by Floyd-Warshall will be eval- uated for significance using eq. 8 and the ones with NFA $\leq \varepsilon$ will be kept (lines 21 to 25 ).

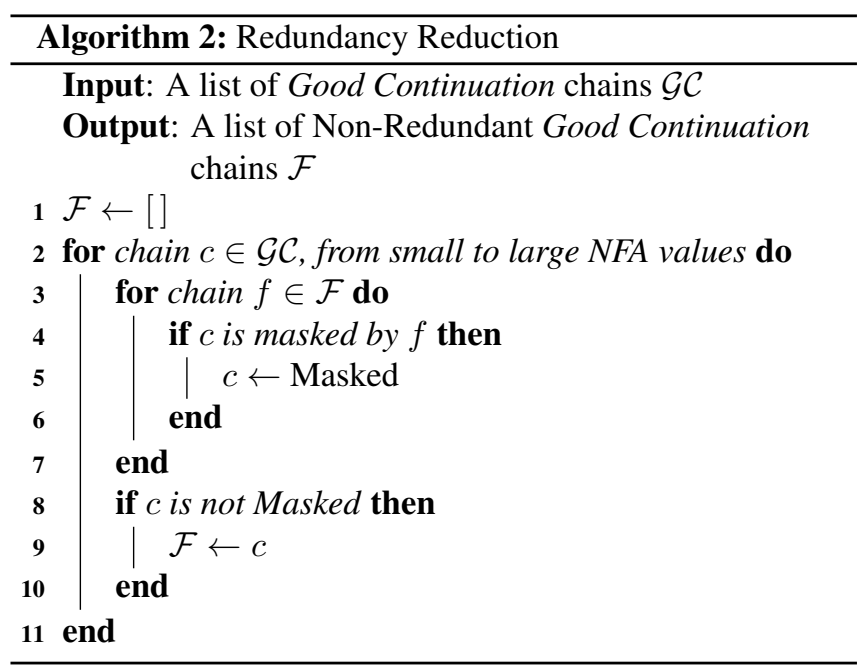

Once all the good continuation events are found, one might be interested in keeping only the maximal meaningful events. Note that one good continuation event might mask another smaller event contained in itself. We say that an event $A$ masks an event $B$, if, when we take the elements of $A$ from $B, B$ is no longer meaningful. Obtaining a list of only the most meaningful events, which are not masked by any other event, can be done by following the simple steps described in Algorithm 2. First, the meaningful chains are ordered by their significance value (lowest NFA first). A second list is created which in the beginning contains only the most meaningful chain. Then, the first list is traversed, checking each chain for maskings with the chains in the second list. If the chain is not masked, it is added to the second list.

\section{EXPERIMENTS}

This section illustrates the proposed algorithm on synthetic and real sets of points. An online demonstration of the method is available and readers are invited to draw or upload a list of $2 \mathrm{D}$ points, as well as to visit the archive of previous experiments. $^{2}$

Figure 3 shows experimental results for the good continuation detector algorithm on dot patterns drawn manually on the online demo. In Fig. 4, we used example data from the signature validation dataset presented in [16]. Each example consists of a pattern of $2 \mathrm{D}$ points that follows the trace of a signature, as it is captured by a drawing tablet. To make the examples more challenging, we also added 100 random points to the original data to create a second dataset.

The examples in the first four rows of Fig. 3 show the ability of the algorithm to cope with background noise. In the

\footnotetext{
${ }^{2}$ http://dev.ipol.im/ jlezama/ipol_demo/lgrm_ good_continuation_icip/ (User: demo Password: demo)
} 


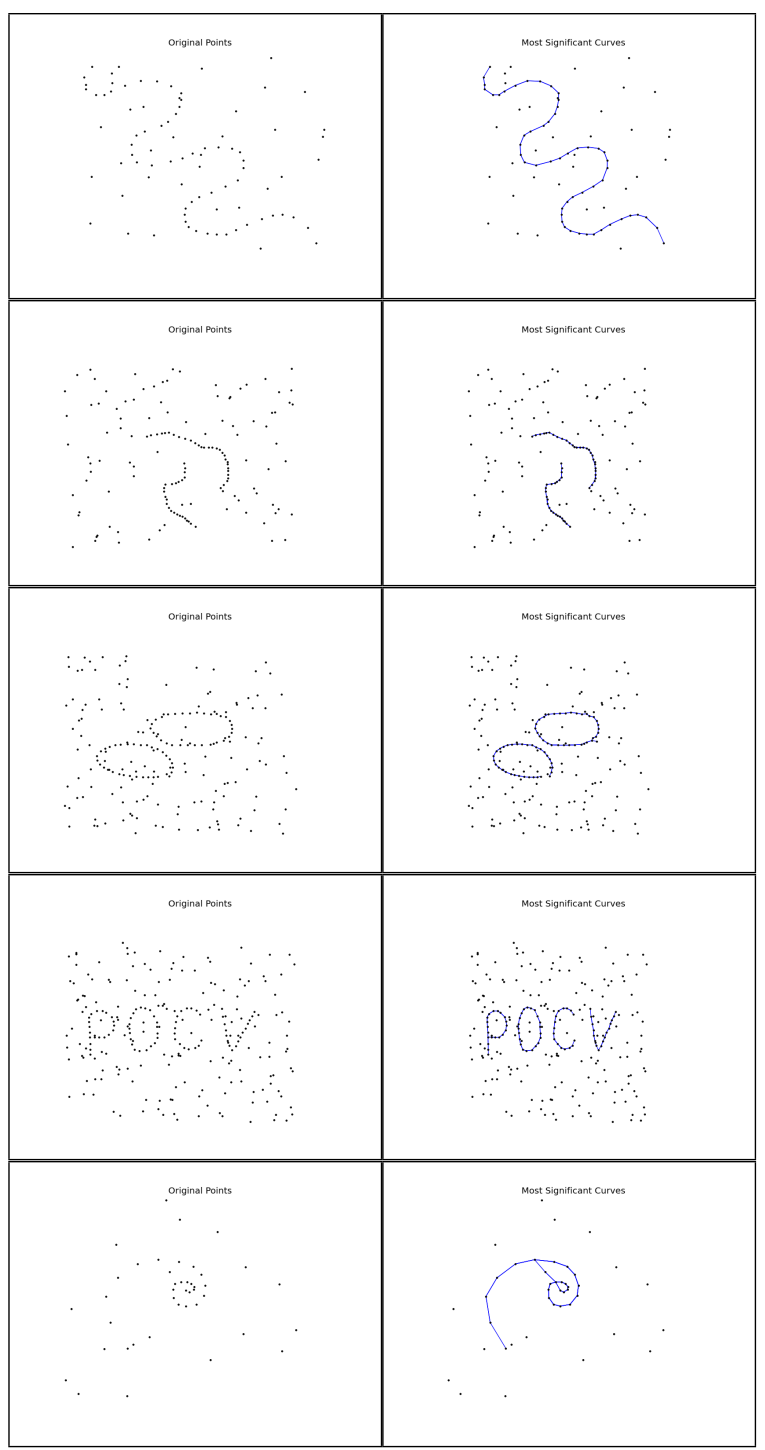

Fig. 3. Experimental results for the good continuation detector. The input examples have been drawn using the online tool companion to this article. Left: original points. Right: most meaningful chains after applying the masking principle.

last row, the algorithm continues the spiral into a path that is not the most perceptually relevant.

The sets of points on Fig. 4 are somehow more challenging. In the first example, some gaps are not completed. This can be explained by the fact that the triplet model is not scale invariant, so more spaced points make triplets more costly. Another source of error is that the paths retrieved by FloydWarshall are suboptimal with respect to the NFA minimization that is intended by the algorithm.

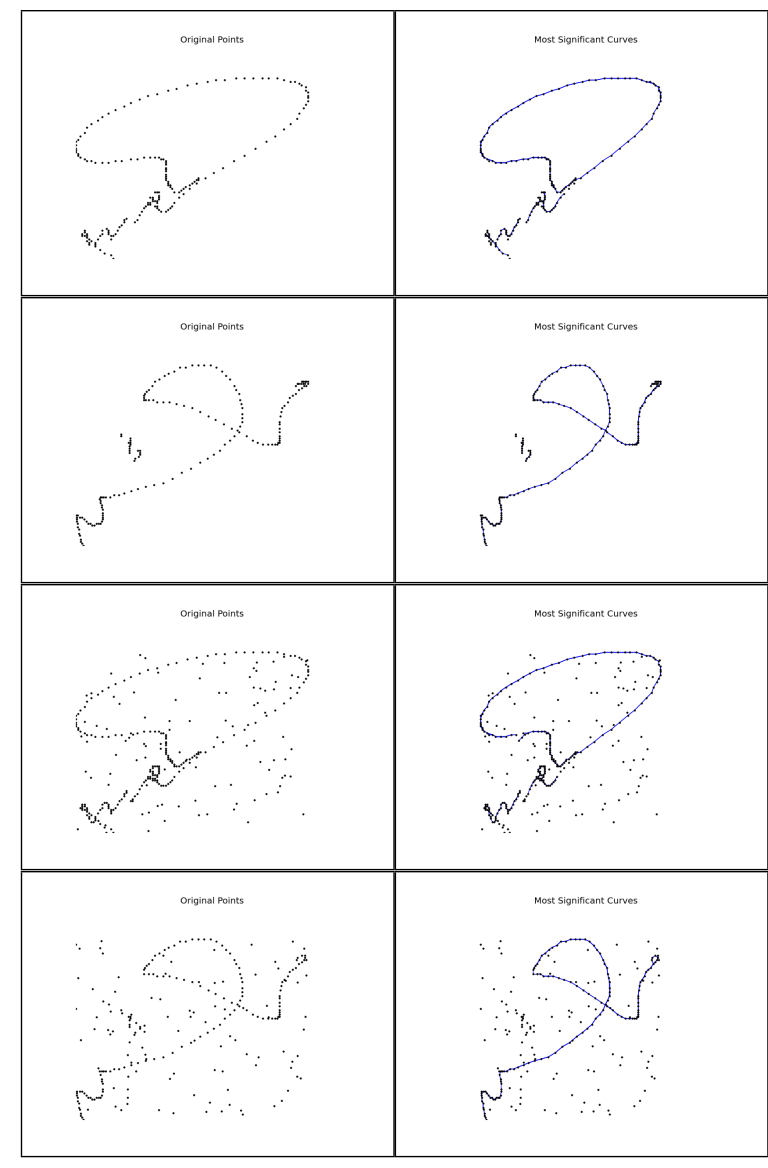

Fig. 4. Algorithm results on signature images from [16]. Left: original points. Right: most meaningful chains after applying the masking principle. In the two bottom rows, we added 100 dots of random noise to the original data. Our algorithm is still able to detect the meaningful configurations of points in good continuation.

\section{CONCLUSION}

We presented an unsupervised algorithm to detect good continuation configurations of points, among a noisy set. The method is robust and produces good results without parameter tuning. The algorithm is able to handle the smoothness of the curve and the regularity of its points. Nevertheless, when both aspects vary rapidly, the algorithm may fail to capture the best interpretation. Future work will concentrate on higher-order models, adding the local curvature in the evaluation, as well as scale invariance.

\section{REFERENCES}

[1] W. Metzger, Gesetze des Sehens, Verlag Waldemar Kramer, Frankfurt am Main, third edition, 1975. 
[2] G. Kanizsa, Organization in vision: Essays on Gestalt perception, Praeger New York:, 1979.

[3] J. Wagemans, J. H. Elder, M. Kubovy, S. E. Palmer, M. A. Peterson, M. Singh, and R. von der Heydt, "A century of gestalt psychology in visual perception: I. perceptual grouping and figureground organization.," Psychological Bulletin, vol. 138, no. 6, pp. 1172-1217, 2012.

[4] J. Wagemans, J. Feldman, S. Gepshtein, R. Kimchi, J. R. Pomerantz, P. A. van der Helm, and C. van Leeuwen, "A century of gestalt psychology in visual perception: II. conceptual and theoretical foundations.," Psychological Bulletin, vol. 138, no. 6, pp. 1218-1252, 2012.

[5] S. E. Palmer, Vision Science: Photons to Phenomenology, The MIT Press, 1999.

[6] D. Lowe, Perceptual Organization and Visual Recognition, Kluwer Academic Publishers, 1985.

[7] Amnon Sha'ashua and Shlmon Ullman, "Structural saliency: The detection of globally salient structures using a locally connected network," in Second International Conference On Computer Vision, 1988, pp. 321327.

[8] G. Guy and G. Medioni, "Perceptual grouping using global saliency-enhancing operators," in ICPR92, 1992, pp. I:99-103.

[9] Leandro A. Loss, George Bebis, Mircea Nicolescu, and Alexei Skourikhine, "Perceptual grouping based on iterative multi-scale tensor voting," in $I S V C$ (2), 2006, pp. 870-881.

[10] Dian Gong and Gérard Medioni, "Probabilistic tensor voting for robust perceptual grouping," in Computer Vision and Pattern Recognition Workshops (CVPRW), 2012 IEEE Computer Society Conference on, 2012, pp. $1-8$.

[11] E. Arias-Castro, D. L. Donoho, and X. Huo, "Adaptive multiscale detection of filamentary structures in a background of uniform random points," 2006.

[12] E. Arias-Castro, D. L. Donoho, X. Huo, and C. A. Tovey, "Connect the dots: How many random points can a regular curve pass through?," 2005.

[13] Z. Pizlo, M. Salach-Golyska, and A. Rosenfeld, "Curve detection in a noisy image," Vision Research, vol. 37, no. 9, pp. 1217-1241, 1997.

[14] A. Desolneux, L. Moisan, and J.M. Morel, From Gestalt Theory to Image Analysis, a Probabilistic Approach, Springer, 2008.
[15] F. Cao, "Application of the Gestalt principles to the detection of good continuations and corners in image level lines," Computing and Visualization in Science, vol. 7, pp. 3-13, 2004.

[16] S. Al-Maadeed, W. Ayouby, A. Hassaine, A. Almejali, A. Al-Yazeedi, and R. Al-Atiya, "Arabic signature verification dataset," in Proceedings of the International Arab Conference on Information Technology, 2012. 